\title{
Mysterious Uškiani - Armenian Gold: The Driving Force for Development in the Bronze Age Caucasus Region?
}

The Gegharkunik region on the south eastern edge of Lake Sevan (Armenia) is a part of southern Caucasia uniquely suited to demonstrate the intensive interchange between pre-Christian settlement and gold mining. The enclosed landscape can be seen in this regard as a prehistoric terra incognita due to the wholesale lack of archaeological and archaeometallurgical investigations to date. It has been proven that there was major exploitation of gold from the defined geographical area in antiquity. One of the largest gold mines in Near East is still located there today. The mines position on the Sotk pass, which is the direct connection between southern and eastern Caucasia and thus has a strategic importance for the entire region, is also of significance. Initial approaches revealed outlines of the prehistoric settlement patterns which could be placed in relation to contemporary gold mining in a clearly delineated natural corridor along this superregional communication and trade route. The intention is to implement an intensive holistic-archaeological and archaeometallurgical investigation of the settlement network in the surroundings of the gold mine and an interdisciplinary attempt to imbed these structures in the larger ecological and anthropogenic environment. The existence of placer gold in several local rivers as well as ancient mining traces has already been demonstrated. The findings of placer gold, along with the geochemical fingerprinting of primary and secondary gold sources from this region will make it possible to analyze the origins of golden artefacts found in the wider vicinity of the area. The opportunity to study gold items from numerous Age finds from Armenia allow for an extensive reconstruction of the early use of gold and its importance in ancient Near Eastern cultures. 